\title{
Air-Coupled Ultrasonic Ferroelectret Receiver with Additional DC Voltage ${ }^{\dagger}$
}

\author{
Mate Gaal 1,*, Rui Caldeira ${ }^{1}$, Jürgen Bartusch ${ }^{1}$ and Mario Kupnik ${ }^{2}$ \\ 1 Bundesanstalt für Materialforschung und-prüfung (BAM), Berlin, Germany; rui.caldeira@bam.de (R.C.); \\ juergen.bartusch@bam.de (J.B.) \\ 2 Technische Universität Darmstadt, Darmstadt, Germany; kupnik@emk.tu-darmstadt.de \\ * Correspondence: mate.gaal@bam.de; Tel.: +49-30-8104-3174 \\ + Presented at the Eurosensors 2017 Conference, Paris, France, 3-6 September 2017.
}

Published: 16 August 2017

\begin{abstract}
Highly sensitive air-coupled ultrasonic sensors are essential for various applications such as testing of composite materials. One of the major challenges for the development of air-coupled ultrasonic sensors is the impedance matching to air. With a lower acoustic impedance than the usual piezoelectric materials, charged cellular polypropylene film (cPP) offers better matching to air with a similar piezoelectric coefficient. The piezoelectric behaviour demonstrated by cPP comes from polarized air cells that create a permanent internal voltage. The sensitivity of the sensor varies with the application of an additional DC bias voltage. Thus, this work presents a cPP ultrasonic sensor with an improvement of up to $15 \pm 1 \mathrm{~dB}$ on the signal-to-noise ratio.
\end{abstract}

Keywords: ultrasonic; air-coupled; cellular polypropylene; transducer; ferroelectret

\section{Introduction}

The impedance matching to air is the main challenge for air-coupled ultrasonic sensors. Ferroelectrets are materials with a low acoustic impedance making them suitable for air-coupled ultrasonic applications. They are a subtype of electrets defined as films of polarized or charged polymer foams, so that the polarization gives them piezoelectric properties. One member of this family is charged cellular polypropylene (cPP) (Figure 1) [1]. It presents a lower acoustic impedance $\left(0.03 \times 10^{6} \mathrm{Ns} / \mathrm{m}^{3}\right)$ than the usual piezoelectric materials used as transducers (e.g., $30 \times 10^{6} \mathrm{Ns} / \mathrm{m}^{3}$ for $\mathrm{PZT}$ ) while exhibiting a similar piezoelectric constant after corona charging (about $200 \mathrm{pC} / \mathrm{N}$ ) [2].

In the process of corona charging the film becomes permanently polarized showing a corresponding internal voltage which makes it applicable as an electret microphone [3]. A force applied to the surface of the film compresses the cells and moves the enclosed charge carriers in relation to each other [2]. If the cPP film is provided with electrodes, the resulting change in internal voltage can be measured as an electric signal proportional to the thickness change of the film. With a resonance frequency in the order of hundreds of $\mathrm{kHz}$, the cPP film is suitable as a sensor and as an actuator for nondestructive ultrasonic testing. Other applications include pickups for musical instruments and accelerometers [4]. Stacking several layers of cPP lowers the resonance frequency of the transducers [5].

In this work, we present a cPP sensor with its sensitivity regulated by the application of an additional DC bias as in a condenser microphone or capacitive micromachined ultrasonic transducer. A DC bias voltage of $2000 \mathrm{~V}$ increases the sensitivity by up to $15 \mathrm{~dB}$ with only $1 \mathrm{~dB}$ increase of the noise, thus broadening the application possibilities of $\mathrm{cPP}$ transducers. 


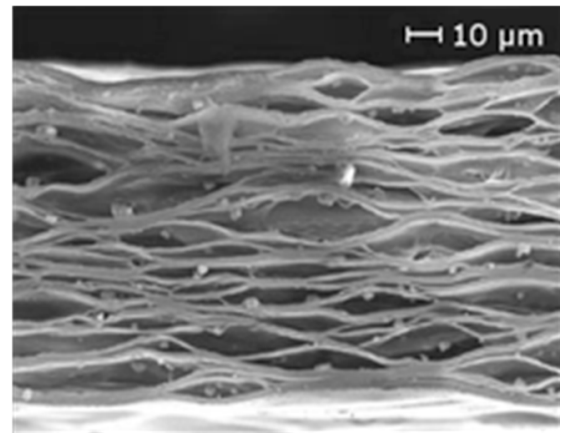

(a)

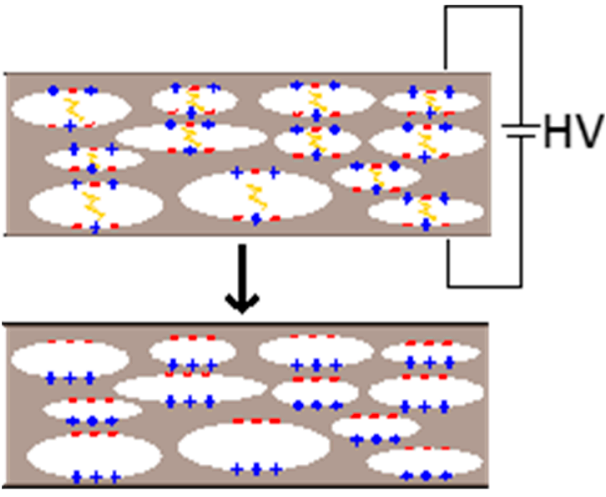

(b)

Figure 1. (a) Cellular polypropylene (cPP) films have an internal structure with voids which provides low acoustic impedance close to that of air. (CEMFIT Ltd.) (b) The film is polarized with corona discharges. The charge is trapped at the surfaces of the voids creating quasi-permanent polarization equivalent to a voltage $V_{0}$.

\section{Materials and Methods}

\subsection{Theoretical Background}

Usually cPP transducers are described within the concept of piezoelectricity. For our purpose, it is interesting to analyze them starting from the functioning principles of capacitive microphones. When a sound wave impinges a capacitive microphone, it changes the distance between the plates and therefore its capacitance. If we apply a bias voltage $V_{\text {bias }}$, the alternating voltage across the capacitor varies due to the change of capacitance. For sufficiently small sound pressure levels the voltage variation is

$$
\Delta V=V_{\text {bias }} \frac{\Delta h}{h}
$$

where $h$ and $\Delta h$ are the distance between the plates and its variation respectively. With electret and specifically ferroelectret microphones, the bias voltage is provided by the internal polarization of the ferroelectret. However, we can increase the sensitivity $\Delta V / \Delta h$ of the ferroelectret receiver by applying an additional external voltage $V_{d c}$, so that we have

$$
\Delta V=\left(V_{0}+V_{d c}\right) \frac{\Delta h}{h}
$$

There is a relation between the internal bias voltage $V_{0}$ and the piezoelectric constant $d_{33}$ :

$$
V_{0}=\frac{c_{33} d_{33} h}{\varepsilon}
$$

where $c_{33}$ is Young modulus in thickness direction, $\varepsilon$ permittivity and $h$ the thickness of the ferroelectret [1].

\subsection{Experimental Setup}

A transducer based on cPP was developed. A low-noise high-voltage module was developed to provide additional DC bias voltage to the $\mathrm{cPP}$ film. It was integrated into a system composed of a previously developed ultrasonic sensor and a pre-amplifier (Figure 2). To evaluate the relation between the bias voltage and the film sensitivity, a through-transmission setup consisting of an ultrasonic transmitter and a DC-biased cPP sensor was used. The amplitude of the received signal was measured in dependence on varying DC voltage while the transmitting signal was kept constant. Transducers with stacked layers were investigated as well. In these transducers, up to four layers of cPP were glued together, in order to increase the thickness of the transducer, and, thus, change its resonance frequency and its internal voltage $V_{0}$. 


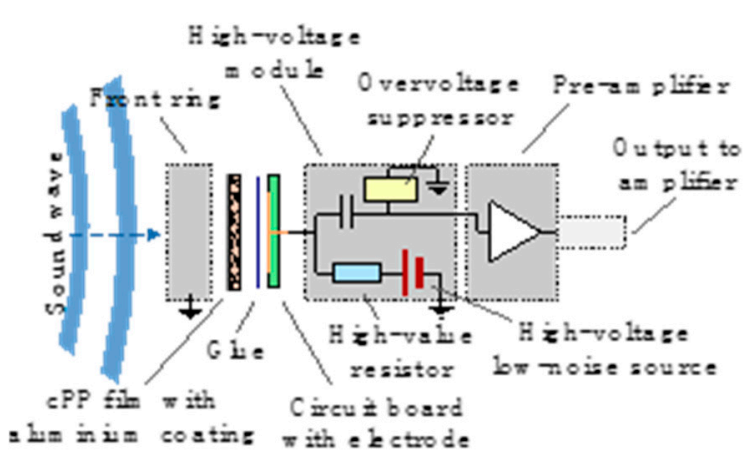

(a)

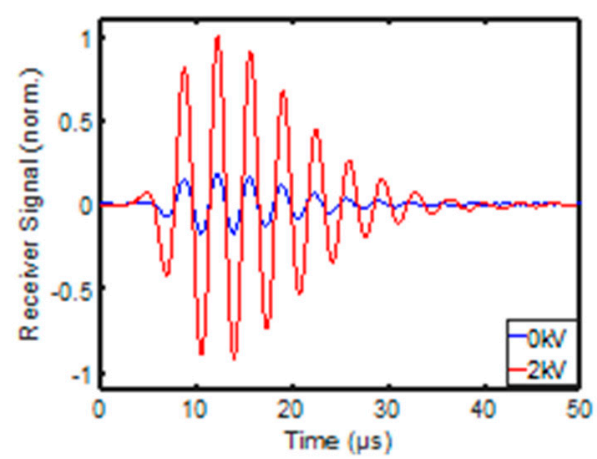

(b)

Figure 2. (a) Receiver with a high-voltage module that adds an external bias-voltage $V_{d c}$ to the existing internal voltage $V_{0}$ of the ferroelectret film. (b) Receiver signals with a bias-voltage $V_{d c}$ of $0 \mathrm{~V}$ (blue) and $2000 \mathrm{~V}$ (red).

\section{Results}

Bias voltage of $2000 \mathrm{~V}$ increased the sensitivity of the single layer sensor by $15 \mathrm{~dB}$. The noise level increased only by $1 \mathrm{~dB}$ (Figure $2 \mathrm{~b}$ ). The amplitude of the received ultrasonic signal depends linearly on the applied bias voltage $V_{d c}$ (Figure 3), as predicted in Equation (2). Applying a bias voltage of the same magnitude as the internal voltage, but of opposite sign, renders the film insensitive. This is used to determine the internal voltage as the intercept of the fitted straight lines. For the single-layer cPP sensor, the internal voltage was $473 \mathrm{~V}$ with $\pm 16 \mathrm{~V}$ tolerance, while for stacked layer sensors the internal voltage grew with the number of layers: for two layers $1261 \pm 25 \mathrm{~V}$, three layers $2186 \pm 29 \mathrm{~V}$, and four layers $3233 \pm 56 \mathrm{~V}$.

Equation (2) shows us that the slopes of the straight lines in Figure 3 are equal to the effective strain of the sensor. The term "effective strain" should be used because of viscoelastic inhomogeneity of $\mathrm{CPP}$ and inhomogeneous sound field so that the measured signal is a result of an integration over the whole sensor aperture. This strain was $369 \pm 4,322 \pm 5,152 \pm 2$ and $75 \pm 1\left[\times 10^{-6}\right]$ for sensors with $1,2,3$ and 4 layers, respectively.

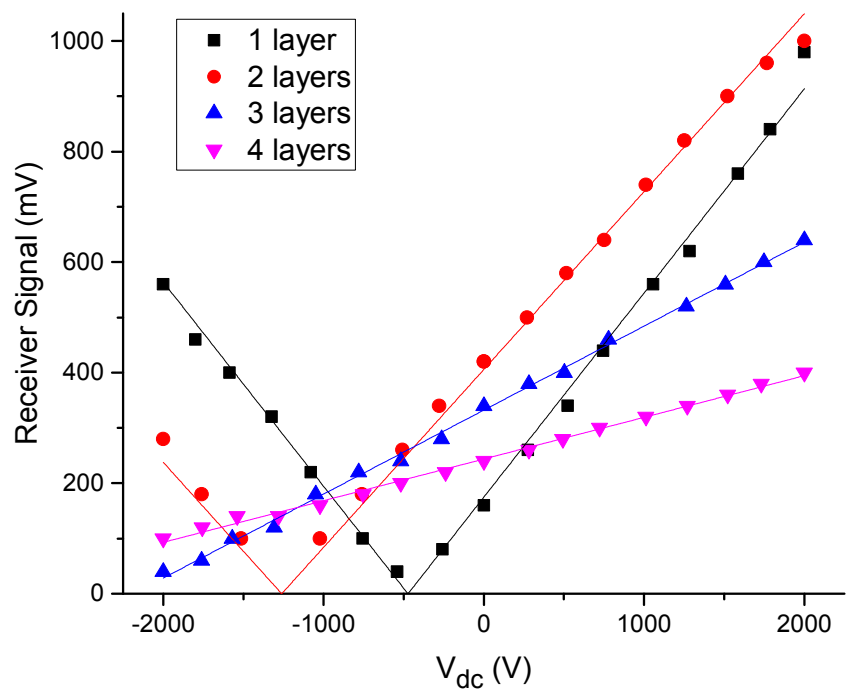

Figure 3. Peak-to-peak signals received with a high-voltage module versus bias-voltage $V_{d c}$.

\section{Discussion}

The internal voltage $V_{0}$ grows faster than proportional with the increasing number of cPP layers. This may indicate that the internal voltage varies from layer to layer within the same transducer. This may be the consequence of electrode deposition, which may alter the properties of the outer layer 
due to thermal load. The reduction of strain with the number of layers has several causes. Not only the construction of the sensor, but also the parameters of the transmitter and the geometrical parameters (e.g., distance) also affect the sound pressure and thus the strain of the receiver. With an increasing number of layers in a sensor, the benefit of using bias voltage $V_{d c}$ decreases, because the internal electric field coming from $V_{d c}$ decreases.

The implementation of $V_{d c}$ leads to the most sensitive ultrasonic transmission system for nondestructive testing. This opens new possibilities for inspection of more difficult components. The determination of internal voltage in ferroelectrets is an application of scientific value. Beyond nondestructive testing, other applications are possible. An example is the construction of extremely sensitive air-coupled ultrasonic receivers.

Acknowledgments: This study was financed by the research program "Menschen, Ideen" of BAM. Our previous work with cPP transducers was supported by programs ZIM (Zentrales Innovationsprogramm Mittelstand) and MNPQ-Transfer (Messen, Normen, Prüfen und Qualitätssicherung) of the German Federal Ministry of Economic Affairs and Energy. We have received no funds for covering the costs to publish in open access.

Conflicts of Interest: The authors declare no conflict of interest.

\section{References}

1. Paajanen, M.; Lekkala, J.; Kirjavainen, K. ElectroMechanical Film (EMFi)-A new multipurpose electret material. Sens. Actuators 2000, 84, 95-102.

2. Hillenbrand, J.; Sessler, G.M. DC-biased ferroelectrets with large piezoelectric d33-coefficients. J. Appl. Phys. 2008, 103, 07413.

3. Wegener, M. Piezoelectric polymer foams: Transducer mechanism and preparation as well as touch-sensor and ultrasonic-transducer properties. Proc. SPIE 2010, 7644, doi:10.1117/12.847245.

4. Hillenbrand, J.; Kodejska, M.; Garcin, Y.; von Seggern, H.; Sessler, G.M. High-sensitivity piezoelectret-film accelerometers. IEEE Trans. Dielectr. Electr. Insul. 2010, 17, doi:10.1109/TDEI.2010.5539670.

5. Hillenbrand, J.; Sessler, G.M. Stacked piezoelectret microphones with of simple design and high sensitivity. IEEE Trans. Dielectr. Electr. Insul. 2006, 13, doi:10.1109/TDEI.2006.247821.

6. Gaal, M.; Bartusch, J.; Dohse, E.; Schadow, F.; Köppe, E. Focusing of Ferroelectret Air-Coupled Ultrasound Transducers. In Proceedings of the Review of Progress in Quantitative Nondestructive Evaluation, Minneapolis, MN, USA, 26-31 July 2015.

(C) 2017 by the authors. Licensee MDPI, Basel, Switzerland. This article is an open access article distributed under the terms and conditions of the Creative Commons Attribution (CC BY) license (http://creativecommons.org/licenses/by/4.0/). 